\title{
Altered susceptibility to apoptosis and $N$-glycan profiles of hematopoietic KG1a cells following co-culture with bone marrow-derived stromal cells under hypoxic conditions
}

\author{
XINGCHEN PANG $^{1 *}$, YI WANG $^{2 *}$, SIJIE ZHANG ${ }^{1}$, ZENGQI TAN ${ }^{3}$, JIA GUO $^{4}$, FENG GUAN $^{1,3}$ and XIANG LI ${ }^{3,5}$ \\ ${ }^{1}$ Key Laboratory of Carbohydrate Chemistry and Biotechnology, Ministry of Education, School of Biotechnology, \\ Jiangnan University, Wuxi, Jiangsu 214122; ${ }^{2}$ Department of Hematology, Provincial People's Hospital, Xi'an, Shaanxi 710068; \\ ${ }^{3}$ Joint International Research Laboratory of Glycobiology and Medicinal Chemistry, College of Life Science, \\ Northwest University, Xi'an, Shaanxi 710069; Institute of Biomedical Engineering and Health Sciences, \\ Changzhou University, Changzhou, Jiangsu 213164; ${ }^{5}$ Wuxi School of Medicine, \\ Jiangnan University, Wuxi, Jiangsu 214122, P.R. China
}

Received December 2, 2017; Accepted June 15, 2018

DOI: $10.3892 /$ or.2018.6548

\begin{abstract}
Mesenchymal stromal cells are an important component of the bone marrow microenvironment (niche), where they support hematopoiesis via direct cell-cell interactions with hematopoietic stem and progenitor cells, and by releasing soluble factors. Glycans, including $N$-glycans, are involved in numerous biological processes, including inflammation, cell-cell interactions, as well as cancer development and progression. Lectin-based microarray analysis has provided a powerful new tool in recent years, for the investigation of aberrantly expressed $\mathrm{N}$-glycans and their functions in the bone marrow microenvironment. In the present study, we used an in vitro stromal/hematopoietic cell co-culture system to examine the effects of stromal-derived signals on apoptosis
\end{abstract}

Correspondence to: Professor Feng Guan, Key Laboratory of Carbohydrate Chemistry and Biotechnology, Ministry of Education, School of Biotechnology, Jiangnan University, 1800 Lihu Avenue, Wuxi, Jiangsu 214122, P.R. China

E-mail: guanfeng@nwu.edu.cn

Professor Xiang Li, Joint International Research Laboratory of Glycobiology and Medicinal Chemistry, College of Life Science, Northwest University, 229 Taibai Road (North), Xi'an, Shaanxi 710069, P.R. China

E-mail: xiangli@nwu.edu.cn

${ }^{*}$ Contributed equally

Abbreviations: HSCs, hematopoietic stem cells; HPCs, hematopoietic progenitor cells; MDS, myelodysplastic syndrome; HIF-1 $\alpha$, hypoxia-inducible factor $1 \alpha$; TNF $\alpha$, tumor necrosis factor $\alpha$; PTMs, post-translational modifications; EMT, epithelialmesenchymal transition; LEF-1, lymphoid enhancer-binding factor-1

Key words: hypoxia, $\mathrm{N}$-glycan, bone marrow microenvironment, stromal cells, co-culture system susceptibility of co-cultured KG1a hematopoietic cells under hypoxic $\left(1 \% \mathrm{O}_{2}\right)$ conditions. MALDI-TOF/TOF-MS analysis was used for the comparative global profiling of $\mathrm{N}$-glycans in KG1a cells and co-cultured KG1a cells under hypoxia. KG1a cells became more susceptible to p53-dependent apoptosis when co-cultured with HS27A human stromal cells (derived from normal bone marrow) under hypoxia. We observed enhanced levels of core-fucosylated $N$-glycans (catalyzed by FUT8), bisecting GlcNAc (catalyzed by MGAT3), and their corresponding genes in co-cultured cells. In addition we observed that overexpressing MGAT3 or FUT8 facilitated cell apoptosis in KG1a cells. Collectively, our data revealed the profiling of $N$-glycans in KGla cells before and after stroma contact. Our findings and future functional studies of core-fucosylated $N$-glycans and bisecting GlcNAc, will improve our understanding of the bone marrow microenvironment.

\section{Introduction}

The bone marrow microenvironment consists of a specialized population of cells that play essential roles in regulation, self-renewal and differentiation of adult stem cells (1-4). The microenvironment supports maturation of hematopoietic stem cells (HSCs) and hematopoietic progenitor cells (HPCs) and their release into the vascular system (5-7). Human bone marrow-derived stromal cell lines HS5 and HS27A, co-cultured with myeloid cells, are frequently used in studies of crosstalk between cells in the bone marrow microenvironment and hematopoietic cells (8-13). For example, HS5 and HS27A have been used to establish a xenotransplantation murine model of myelodysplastic syndromes (MDS), a group of clonal hematopoietic disorders characterized by dysregulation of programmed cell death (apoptosis) and ineffective hematopoiesis in both normal and clonal (transformed) hematopoietic cells $(12,14)$. Kerbauy et al $(14)$ observed engraftment of distinct clonal MDS-derived hematopoietic precursors when stromal cells (HS5 and HS27A combined) were co-injected via an intramedullary route. Our previous study found that 
intravenous co-administration of HS27A cells (but not HS5 cells) with HPCs from MDS patients facilitated engraftment of clonal $\mathrm{CD}_{3} 4^{+}$cells of any karyotype (12), indicating that HS27A cells were more effective than HS5 in supporting primitive clonal MDS precursors (12).

Glycans, a major category of biomolecules, are commonly attached to proteins and lipids to form glycoproteins, glycolipids, glycosaminoglycans and other types of glycoconjugates (15-17). Glycans play key roles in a variety of biological processes, including cell adhesion, molecular trafficking, receptor activation and signal transduction $(17,18)$. They are also involved in malignant hematopoiesis. $\mathrm{CoCl}_{2}$ (hypoxia mimic) induced expression of cell surface glycans recognized by both $\beta$-galactoside- and GlcNAc-binding lectins in HL-60 cells (19). Galectin-3 is developmentally expressed in human myeloid cells, and is strongly upregulated on the surface of late mature myeloid cells (20). Conversely, it also promotes proliferation and angiogenesis of endothelial cells differentiated from bone marrow mesenchymal stem cells (21). An important characteristic of the HSC microenvironment is that low oxygen $\left(\mathrm{O}_{2}\right)$ tension and hypoxia (defined as $\mathrm{O}_{2}$ tension $<2 \%$ ) is essential for HSC function (22-25). In vitro $\mathrm{BM}$ cells are always cultured under hypoxic conditions in order to maintain their primitive phenotype and self-renewal ability (26). In addition, the key regulator of cellular adaptation to hypoxic stress, hypoxia-inducible factor $1 \alpha(\mathrm{HIF}-1 \alpha)$ can be stabilized under hypoxia $(25,26)$. No study to date has included global analysis of the expression of $N$-glycan in hematopoietic cells co-cultured with vs. without stromal cells under hypoxia.

An in vitro model in which KG1a hematopoietic cells were co-cultured with HS27A cells under hypoxia conditions $\left(1 \% \mathrm{O}_{2}\right)$ was used in the present study. We examined the apoptosis rate of the KGla cells, and the related apoptotic pathway. $N$-glycans linked to glycoproteins were released by PNGase $\mathrm{F}$ and analyzed by MALDI-TOF/TOF-MS, and selectively altered $N$-glycans were further analyzed by lectin staining. By presenting global analysis of patterns of altered $\mathrm{N}$-glycans in hematopoietic cells before and after stroma contact under hypoxia, the present study provides valuable information for future investigations of the bone marrow microenvironment.

\section{Materials and methods}

Cell culture. KG1a cells (derived from acute myeloid leukemia) and bone marrow-derived stromal cell line HS27A are generous gifts from Professor H. Joachim Deeg (Fred-Hutchinson Cancer Research Center, Seattle, WA, USA). KG1a and HS27A were grown, propagated and subjected to experiments between passages 8 and 24, as previously described (27). Multiple aliquots from early passages were cryopreserved for later use.

Total protein extraction. Cells were harvested and lysed with T-PER Tissue Protein Extraction reagent (Thermo Fisher Scientific, Hudson, NH, USA) according to the manufacturer's protocol. In brief, cells were trypsinized, resuspended in phosphate-buffered saline (PBS), added with T-PER reagent containing $1 \%$ protease inhibitor cocktail
(Biotool Ltd., Houston, TX, USA), incubated on ice for $30 \mathrm{~min}$ and homogenized. The sample was centrifuged for $15 \mathrm{~min}$ at $13,000 \times \mathrm{g}\left(4^{\circ} \mathrm{C}\right)$, and the supernatant was collected and stored at $-80^{\circ} \mathrm{C}$.

Enzyme-linked immunosorbent assay (ELISA). Concentrations of secreted tumor necrosis factor $\alpha(\mathrm{TNF} \alpha)$ in culture supernatants were determined (in triplicate) using an ELISA kit (R\&D Systems, Minneapolis, MN, USA) for human TNF $\alpha$ (detection limit $0.5 \mathrm{pg} / \mathrm{ml}$ ). Color intensity of the chromogenic reaction was determined at $490 \mathrm{~nm}$ by a plate reader (Bio-Rad Laboratories, Hercules, CA, USA).

Western blot analysis. Proteins from each sample were separated by $10 \%$ SDS-PAGE and transferred onto polyvinylidene fluoride (PVDF) membranes using the Trans-Blot Turbo Transfer System (Bio-Rad Laboratories). Membranes were soaked in $5 \%(\mathrm{w} / \mathrm{v})$ skim milk in TBST $(20 \mathrm{mM}$ Tris- $\mathrm{HCl}, 150 \mathrm{mM} \mathrm{NaCl}, 0.05 \%$ Tween-20, $\mathrm{pH} \mathrm{8.0)} \mathrm{for} 2 \mathrm{~h}$ at $37^{\circ} \mathrm{C}$, probed with primary antibodies against HIF- $1 \alpha$ (1:1,000; cat. no. 3716; Cell Signaling Technology, Inc., Beverly, MA, USA), p53 (1:1,000; cat. no. 2524; Cell Signaling Technology, Inc.), Bax (1:1,000; cat. no. 2774; Cell Signaling Technology, Inc.), Bcl-2 (1:1,000; 2872; Cell Signaling Technology, Inc.), caspase-3 (1:1,000; cat. no. 9662; Cell Signaling Technology,Inc.),caspase-8(1:1,000; cat.no.9746;Cell Signaling Technology, Inc.), caspase-9 (1:1,000; cat. no. 9502; Cell Signaling Technology, Inc.), NF- $\mathrm{BB}$ (1:1,000; cat. no. 8242; Cell Signaling Technology, Inc.), phospho-NF-кB (1:1,000; cat. no. 3031; Cell Signaling Technology, Inc.) and $\beta$-tubulin (1:5,000; cat. no. T7816; Sigma-Aldrich; Merck KGaA, Darmstadt, Germany) overnight at $4^{\circ} \mathrm{C}$, and incubated with the appropriate HRP-conjugated secondary antibody (1:5,000; cat. nos. A0208 and A0216; Beyotime Institute of Biotechnology, Shanghai, China). Bands were visualized using enhanced chemiluminescence detection kit Westar Nova (Cyanagen Srl, Bologna, Italy) with imaging by ChemiDoc ${ }^{\mathrm{TM}} \mathrm{XRS}+(\mathrm{Bio}-\mathrm{Rad}$ Laboratories).

Cell sorting. KG1a cells were co-cultured with HS27A cells for $24 \mathrm{~h}$ and added together with $\mathrm{CD} 45^{+}$magnetic beads. KG1a cells were isolated by magnetic-activated cell sorting (Miltenyi Biotec, Bergisch Gladbach, Germany) according to the manufacturer's protocol. Cell purity was assessed by staining with fluorescent-tagged anti-CD45 antibody and quantified by flow cytometry with the purity of sorted cells reaching $>95 \%$. Apoptosis was assessed by flow cytometry.

Caspase activity assay. Caspase activity assay was performed according to the manufacturer's instructions (Beyotime Institute of Biotechnology). Briefly, after treatment, isolated KG1a cells were lysed with lysis buffer and the supernatants were incubated with Ac-DEVD-pNA $(200 \mu \mathrm{M})$ substrate for caspase-3 and Ac-IETD-pNA $(200 \mu \mathrm{M})$ substrate for caspase-8, respectively. The reaction was assessed with a plate reader at $405 \mathrm{~nm}$ wavelength.

Quantitative real-time PCR. Total RNA was extracted as previously described (28). Primers were designed using the Primer-BLAST program (https://www.ncbi.nlm.nih. 
Table I. Quantitative real-time PCR primer sequences.

\begin{tabular}{lc}
\hline Genes & \multicolumn{1}{c}{ Primer sequences } \\
\hline FUT8 & F: 5'-TGTCCTGTACTTCATGCGCT-3' \\
RGAT2 & R: 3'-TCCATGACCCTAATGGTCTTTT-5' \\
& F: 5'-AAAGAACACCTGCAGAACCG-3' \\
RGAT3 & F: 3'-GGAATTGACAACGTCCTCGT-5' \\
& R: 3'-TTGCTGAGACCCAGCGG-5' \\
MGAT4B & F: 5'-TCACTGCCGAAGTGTACTGTG-3' \\
& R: 3'-CTGACACTCTGCACTCGCTC-5' \\
MGAT5 & F: 5'-GTGAGGGTAGCCGTCCATAG-3' \\
& R: 3'-CAGCTTGGTTGCACTTGAGA-5'
\end{tabular}

F, forward; R, reverse.

gov/tools/primer-blast/). First-strand cDNA was synthesized from total RNA using a OneScript Plus cDNA Synthesis kit (Abm Canada, Milton, ON, Canada). Quantitative real-time PCR was performed by SYBR-Green I dye detection with BrightGreen Express 2X qPCR Master Mix (Abm Canada). Gene expression was quantified by the $2^{-\Delta \Delta C q}$ method (29). All primer sequences are provided in Table I.

Apoptosis assay. Apoptosis of hematopoietic cells was assayed by Annexin V staining. KG1a cells were cultured under hypoxia for $24 \mathrm{~h}$ with or without HS27A stroma contact, on the basis of our previous studies $(9,11)$. Twelve-well plates were centrifuged at 1,200 rpm (300 x g), the supernatant was discarded, and cells in each well were washed with cold PBS containing $2 \%$ bovine serum albumin (BSA) and assayed using an Annexin V-fluorescein isothiocyanate (FITC) apoptosis kit (Beyotime Institute of Biotechnology). Cells were then labeled with Allophycocyanin (APC)-conjugated anti-CD45 antibody (1:20; cat. no. 368512; BioLegend, San Diego, CA, USA) to distinguish hematopoietic cells (myeloid cells, CD45 ${ }^{+}$) from stromal (CD45), and apoptosis was assayed by flow cytometry (model BD Accuri C6; BD Biosciences, San Jose, CA, USA) using Annexin V-FITC and propidium iodide (PI).

$N$-glycan mass profiling. $\mathrm{N}$-glycans were separated as described in our previous study (30). In brief, we used a size-exclusion spin ultrafiltration unit (Amicon Ultra-0.5 10 KD; EMD Millipore; Merck KGaA, Darmstadt, Germany) to concentrate $2 \mathrm{mg}$ total proteins. Proteins were denatured, centrifuged and then further digested with PNGase F (New England BioLabs, Ipswich, MA, USA) overnight at $37^{\circ} \mathrm{C}$. Released $\mathrm{N}$-glycans were collected and desalted using Sepharose 4B (Sigma-Aldrich; Merck KGaA, Darmstadt, Germany), as previously described (31), then characterized by MALDI-TOF/TOF-MS (ultrafleXtreme; Bruker Daltonik GmbH, Bremen, Germany) in positive-ion mode. Data were analyzed using the FlexAnalysis software program (Bruker Daltonik $\mathrm{GmbH}$ ) and annotated using the GlycoWorkbench software program (http://code.google.com/p/ glycoworkbench/). The stability of MS was estimated using coefficient of variation (CV) percentages based on relative intensity values. The relative variation was calculated by dividing the amount of a given type of $\mathrm{N}$-glycan by the total $N$-glycan amount (30).

Flow cytometric (FACS) analysis of lectin binding affinity. Cells were harvested to a single cell suspension with trypsin or $0.2 \%$ EDTA, and washed three times with PBS. Aliquots $\left(10^{6}\right.$ cells $)$ were resuspended in $100 \mu \mathrm{l}$ diluting Cy3-conjugated lectin (cat. no. L-1000, L-1020, L-1040 and L-1120; Vector Labs, Peterborough, UK) and APC-conjugated anti-human CD45 antibody (1:20; cat. no. 368512; BioLegend) in $0.1 \% \mathrm{BSA} / \mathrm{PBS}$, and incubated on ice for $30 \mathrm{~min}$ in the dark. Lectins were used at a final concentration $100 \mu \mathrm{g} / \mathrm{ml}$. Cells were washed twice with $1 \mathrm{ml}$ PBS and analyzed by flow cytometry as above-mentioned.

Lectin staining. Lectin staining was performed as previously described (30). Cells were spun onto a microscope slide for $5 \mathrm{~min}$ at $300 \mathrm{x} \mathrm{g}$, immobilized with $2 \%$ fresh paraformaldehyde for $15 \mathrm{~min}$ at room temperature, and blocked with $5 \% \mathrm{BSA}$ in 1X PBS for $1 \mathrm{~h}$ at $37^{\circ} \mathrm{C}$. Fixed cells were incubated with $20 \mu \mathrm{g} / \mathrm{ml}$ Cy3-conjugated lectin (LCA, PHA-E) in 5\% BSA for $3 \mathrm{~h}$ in the dark, washed with 1X PBS, stained with $20 \mu \mathrm{g} / \mathrm{ml}$ DAPI in 1X PBS for $10 \mathrm{~min}$, washed again with $1 \mathrm{X}$ PBS, and images were captured with a fluorescence microscope (model Eclipse E600; Nikon, Tokyo, Japan) at the same exposure time and gain factor.

Statistical analysis. Data are presented as the mean \pm SEM. Statistical significance of differences between the means of two groups was evaluated by Student's t-test. Multiple group comparisons were evaluated by ANOVA with Bonferroni's post hoc test.

\section{Results}

Co-culture with stromal cells enhances susceptibility of KGla cells to apoptosis under a hypoxic condition. Hematopoietic cell line KG1a is resistant to apoptosis $(32,33)$. However, apoptosis of KG1a was significantly increased when they were co-cultured with HS27A stromal cells under a hypoxic condition (Fig. 1A and B). We investigated the apoptosis of KG1a cells before and after co-culture with HS27A in the presence of $100 \mu \mathrm{M} \mathrm{CoCl}{ }_{2}$. Treatment with $\mathrm{CoCl}_{2}$ mimics hypoxic conditions by inhibiting prolyl-4-hydroxylases involved in the degradation of HIF-1 $\alpha(34,35) . \mathrm{CoCl}_{2}$ was used as a positive control in the present study. Apoptosis of KGla cells co-cultured with $\mathrm{HS} 27 \mathrm{~A}$ cells was also increased by $\mathrm{CoCl}_{2}$ treatment (Fig. 1C and D). Concentration of $\mathrm{TNF} \alpha$, another factor that enhances susceptibility of KG1a cells to apoptosis, was increased in the co-culture system under hypoxic conditions (Fig. 1E). TNF receptor I (TNFR1) was also increased in KGla cells following co-culture with stromal cells under hypoxic conditions (Fig. $1 \mathrm{~F}$ and $\mathrm{G}$ ).

Hypoxia induces the p53-dependent pathway in co-cultured KGla cells. Our previous studies demonstrated upregulation of p53 levels in hematopoietic cells and primary MDS marrow cells in stromal contact culture $(8,9)$. To investigate the possible 
A

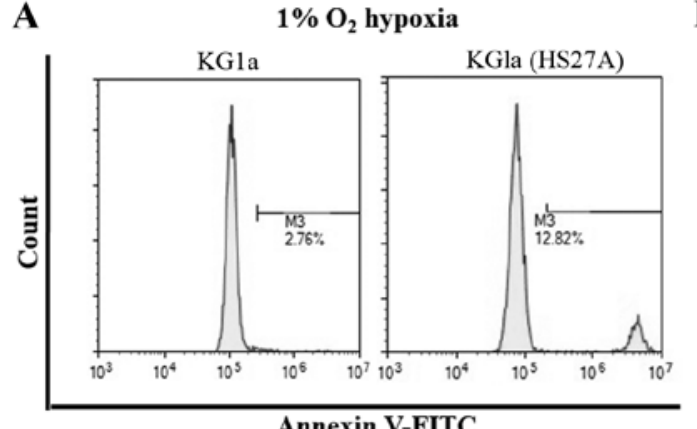

C

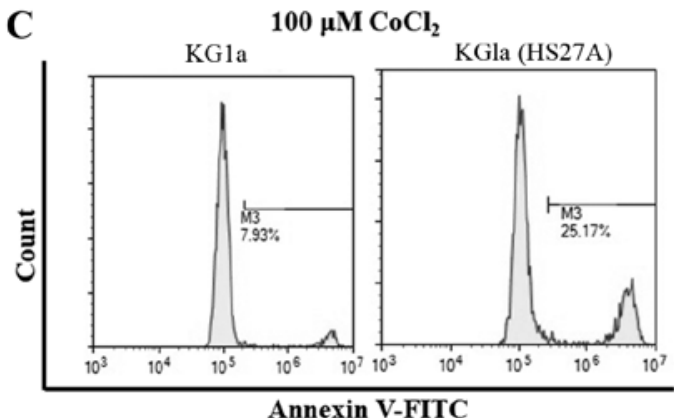

B

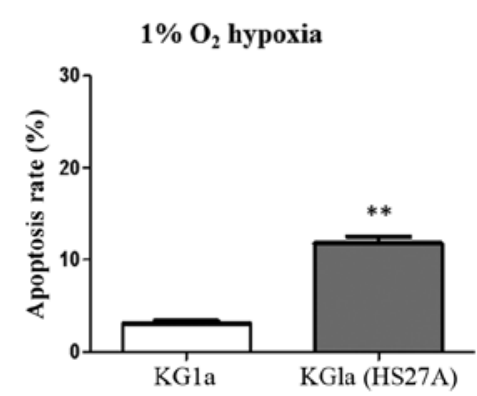

D

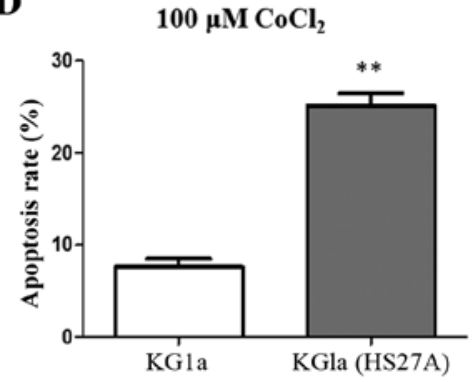

E
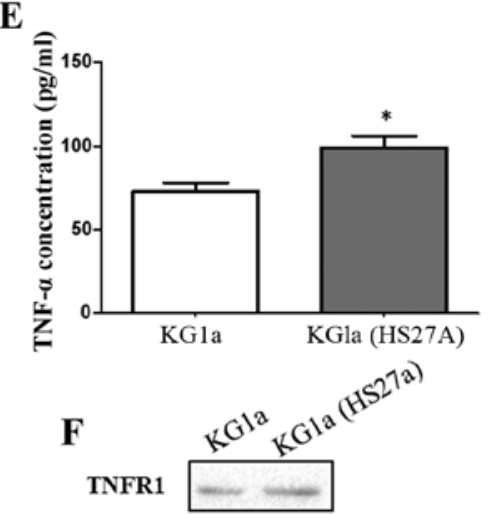

G

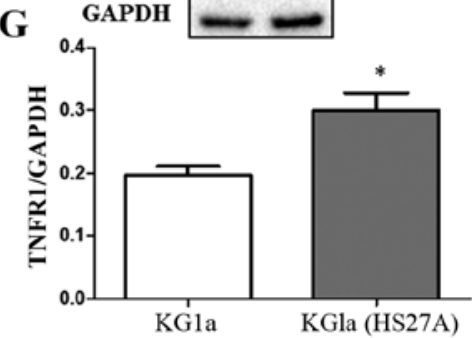

Figure 1. Apoptosis of KG1a cells with and without co-culture with HS27A cells under hypoxic conditions. (A) Flow cytometric (FACS) analysis of the apoptosis rate of KG1a cells alone and KG1a cells co-cultured with HS27A cells under hypoxic $\left(1 \% \mathrm{O}_{2}\right)$ conditions. KG1a cells were sorted using CD45 magnetic beads. Apoptosis rate was assessed by Annexin V staining. (B) Quantification of the apoptosis rate of cells in panel A. (C) Flow cytometric analysis of the apoptosis rate of KGla cells and co-cultured KGla cells under $100 \mu \mathrm{M} \mathrm{CoCl}_{2}$ culture condition. Procedures were performed as described in panel A. (D) Quantification of the apoptosis rate of cells in panel C. (E) TNF- $\alpha$ concentration, assessed by ELISA, in culture medium secreted by KG1a cells and co-cultured KG1a cells. (F) Western blot analysis of TNFR1 in KGla cells and co-cultured KG1a cells. (G) Quantification of TNFR1 expression in KG1a cells and co-cultured KG1a cells. The data shown are representative of three independent experiments. ${ }^{*} \mathrm{P}<0.05,{ }^{* *} \mathrm{P}<0.01$. Error bars, SEM. KG1a (HS27A), co-cultured cells.

involvement of a p53-dependent pathway in hypoxia-induced apoptosis in co-cultured KG1a cells, we examined expression of p53, caspase-3, caspase-8, caspase- 9 and NF- $\mathrm{NB}$ in KG1a cells after co-culture with HS27A cells under hypoxic conditions and $\mathrm{CoCl}_{2}$ treatment. In co-cultured KGla cells, p53, caspase-8, caspase-9 and phosphorylated NF- $\kappa$ B levels were elevated and caspase- 3 and caspase- 8 were cleaved and active (Fig. 2A and B). We further observed that the activities of caspase- 3 and caspase- 8 were also elevated in co-cultured KG1a cells, which appeared to be relevant with the increase in TNF- $\alpha$ and TNFR1 (Fig. 2C). Bax and Bcl-2 levels were respectively upregulated and downregulated in the co-cultured cells, as expected since their expression was affected by $\mathrm{p} 53$ through transcriptional regulation $(36,37)$.

MALDI-TOF/TOF-MS profiles of N-glycans in KGla cells before and after co-culture under hypoxic conditions. $N$-glycans are involved in a variety of biological processes, including cell-cell and cell-matrix interactions, receptor-mediated functions and specific protein functions. Many types of tumor cells display aberrant $N$-glycosylation patterns, and cancer-associated $N$-glycans are potential biomarkers for early detection and diagnosis of cancer (38). We compared MALDI-TOF/TOF-MS profiles of total $N$-glycans in KG1a cells before vs. after co-culture with HS27A cells under hypoxic conditions. MALDI-TOF/TOF-MS spectra of $N$-glycans with signal-to-noise ratios $>5$ from total glycoproteins were annotated using the GlycoWorkbench program (Fig. 3). Eight distinctive $N$-glycans were observed in KGla cells before vs. after co-culture, at m/z 1257.476, 1419.534, 1485.526, 1581.592,
$1688.598,1743.650,1905.711$ and 2067.687 , corresponding to eight proposed $\mathrm{N}$-glycan structures including high-mannose, bi-antennary and tri-antennary types (Fig. 4). There were eight $\mathrm{N}$-glycan structures found in both KGla cells and co-cultured KGla cells, two structures unique to co-cultured KG1a cells, and one structure unique to KG1a cells. Co-cultured KG1a cells showed enhancement of two complex type $N$-glycan structures with fucosylation.

Validation by real-time PCR of N-glycan biosynthesis-related genes. On the basis of our MS data, we examined the expression of $\mathrm{N}$-glycan biosynthesis-related genes by real-time PCR. The glycosyltransferase genes involved in the synthesis of the $\mathrm{N}$-glycan structures listed in Fig. 4 are $\alpha 1,6$-fucosyltransferase (FUT8) and $\beta 1,4-\mathrm{N}-\mathrm{a}$ cetylglucosaminyltransferases $2,3,4 \mathrm{~A}, 4 \mathrm{~B}$ and 5 (MGAT2, MGAT3, MGAT4A, MGAT4B and MGAT5) (Fig. 5A). Among these six genes, FUT8, MGAT3 and MGAT5 showed increased expression in co-cultured KG1a cells, whereas the expression of MGAT2 and $M G A T 4 B$ was not notably altered. These findings were consistent with our MS results. We then applied flow cytometric (FACS) analysis with specific lectins to further examine differentially expressed $\mathrm{N}$-glycans in KG1a cells co-cultured with HS27A cells under hypoxic conditions. Co-cultured cells showed increased expression of $N$-glycan structures recognized by PHA-E (i.e., bisecting GlcNAc structures), LCA (core Fuc $\alpha 1-6 \mathrm{GlcNAc}$ ) and WGA (multivalent Sia and (GlcNAc) $)_{n}$ structures), and reduced expression of structures recognized by ConA (terminal mannose) (Fig. 5B-D). 
A

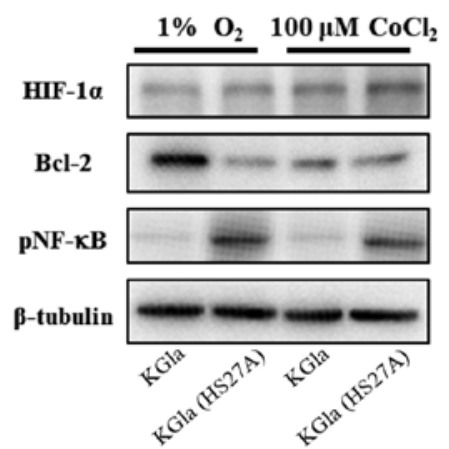

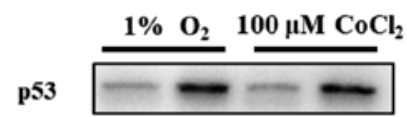

Bax

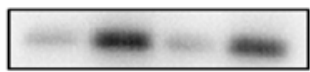

NF- $K B$

GAPDH
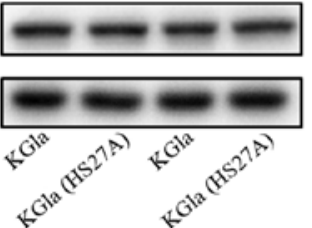

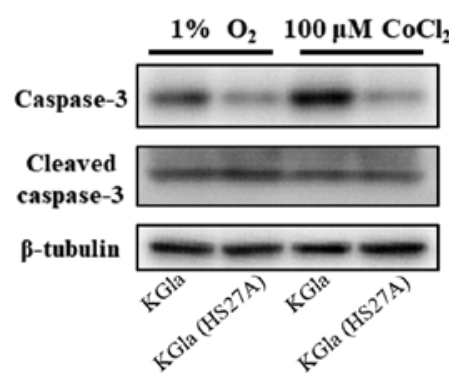

B Cleaved caspase-3

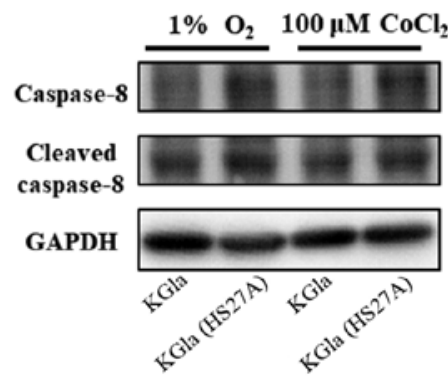

Cleaved caspase-8

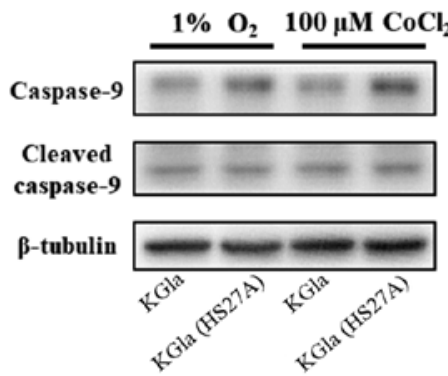

Cleaved caspase-9

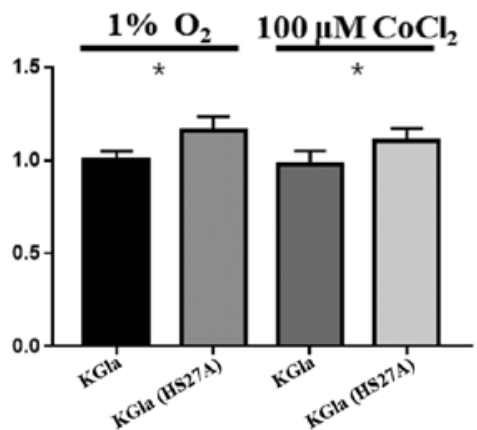

Total caspase-3

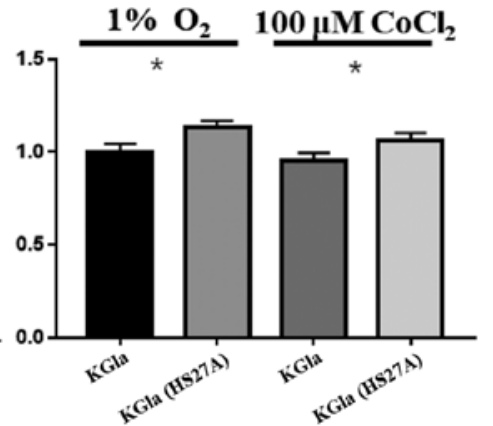

Total caspase-8

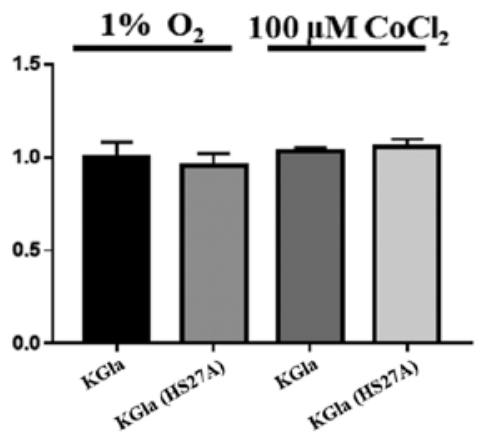

Total caspase-9
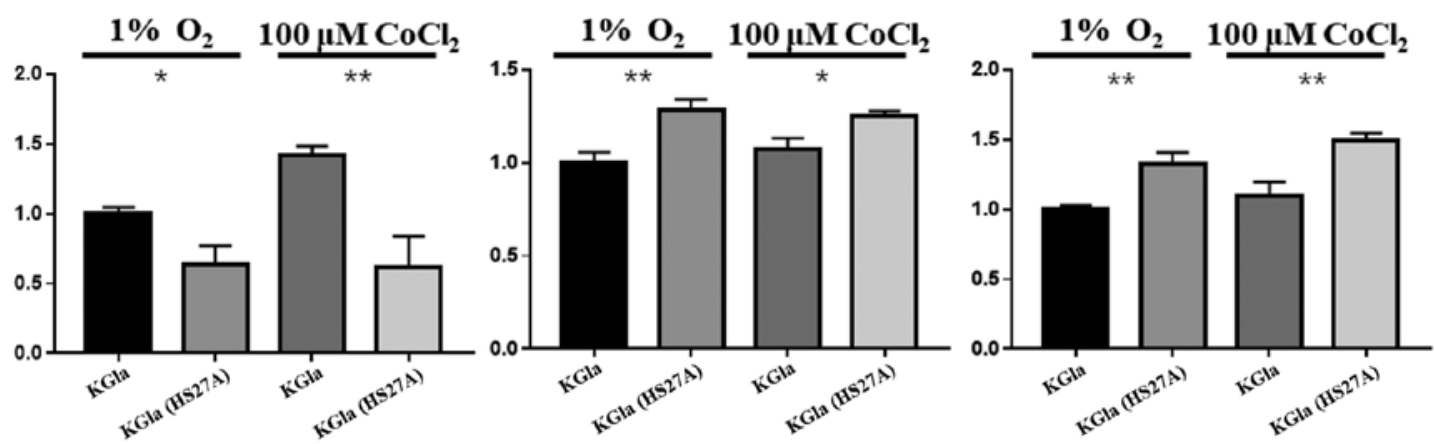

C
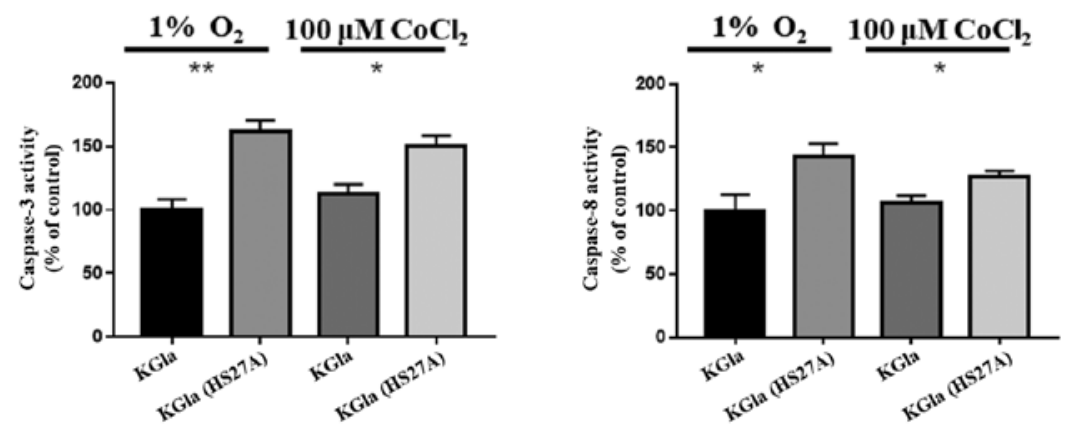

Figure 2. Protein expression in KG1a cells and co-cultured KG1a cells under hypoxia. (A) Protein lysates from KG1a cells and co-cultured KG1a cells under $1 \% \mathrm{O}_{2}$ hypoxia or $100 \mu \mathrm{M} \mathrm{CoCl}_{2}$ conditions were separated on 4 to $12 \%$ Bis-Tris gels, and immunoblotted with antibodies against $\mathrm{HIF}-1 \alpha$, p53, Bax, Bcl-2,

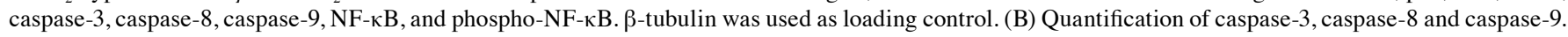
(C) Activity assay of caspase-3 and caspase- 8 . The data shown are representative of three independent experiments. ${ }^{*} \mathrm{P}<0.05$, ${ }^{* *} \mathrm{P}<0.01$. KG1a $(\mathrm{HS} 27 \mathrm{~A})$, co-cultured cells. 

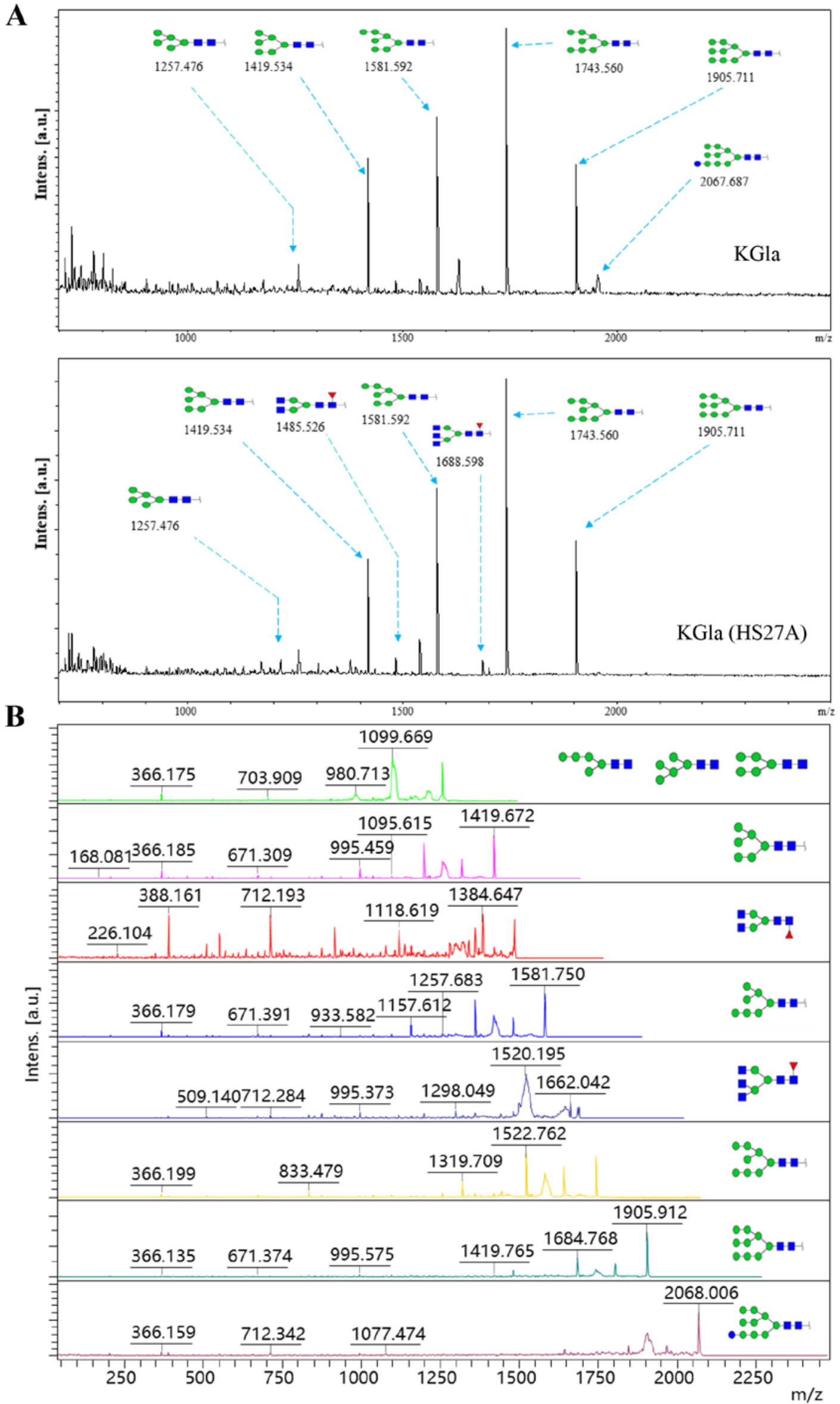

Figure 3. (A) MALDI-TOF/TOF-MS spectra and (B) corresponding MS/MS spectra of $N$-glycans from KGla cells and co-cultured KGla cells under hypoxia. Representative spectra from triplicate experiments are shown. Detailed glycan structures were analyzed using the GlycoWorkbench program. Proposed structures and their m/z values are shown for each peak. KG1a (HS27A), co-cultured cells. 


\begin{tabular}{|c|c|c|c|c|}
\hline \multirow{3}{*}{$\begin{array}{c}\text { Item } \\
1\end{array}$} & \multirow{3}{*}{$\begin{array}{c}\begin{array}{c}\text { Calculated } \\
\mathbf{m} / \mathbf{z}\end{array} \\
1257.476\end{array}$} & \multirow{2}{*}{$N$-glycan structure } & \multicolumn{2}{|c|}{ Relative intensity (CV\%) } \\
\hline & & & KGla & KGla (HS27A) \\
\hline & & - & $0.030(31 \%)$ & $0.023(73 \%)$ \\
\hline 2 & 1419.534 & $\sin _{0 \rightarrow 1}=$ & $0.169(13 \%)$ & $0.157(1 \%)$ \\
\hline 3 & 1485.526 & 19001 & ND & $0.048(34 \%)$ \\
\hline 4 & 1581.592 & + & $0.233(5 \%)$ & $0.274(11 \%)$ \\
\hline 5 & 1688.598 & :00 & ND & $0.017(84 \%)$ \\
\hline 6 & 1743.650 & $\operatorname{lom}_{0 \rightarrow-1}$ & $0.365(5 \%)$ & $0.340(10 \%)$ \\
\hline 7 & 1905.711 & $\because 30=$ & $0.196(8 \%)$ & $0.140(14 \%)$ \\
\hline 8 & 2067.687 & $\because 30 \times$ & $0.010(48 \%)$ & ND \\
\hline
\end{tabular}

Figure 4. Proposed structures and their molecular ions in MALDI-TOF/TOF-MS spectra of $N$-glycans in KG1a before and after co-culture with HS27A cells. KG1a (HS27A), co-cultured cells.

\section{A}

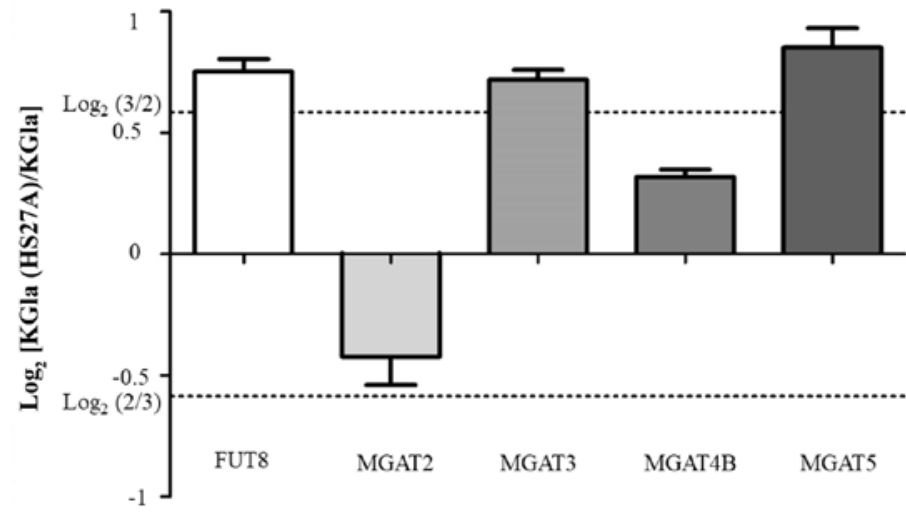

B

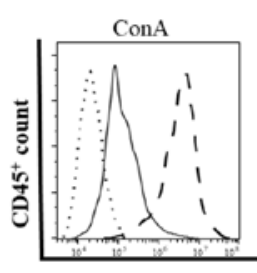

C

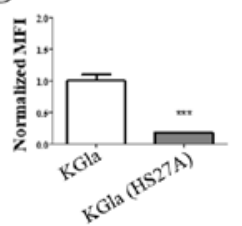

KGla (HS27A)

- - - KGla

.......... Negative control

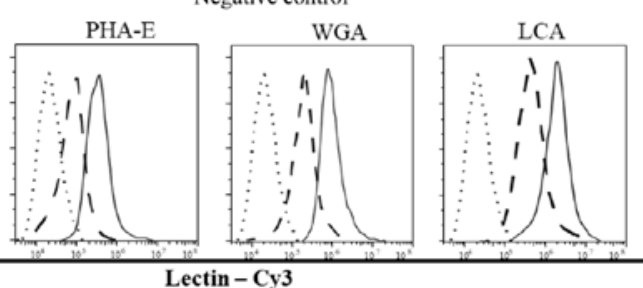

Lectin $-\mathrm{Cy} 3$

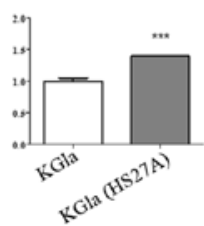

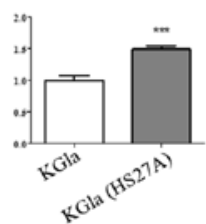

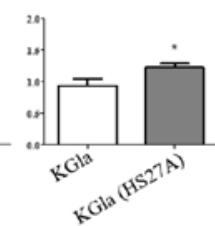

D
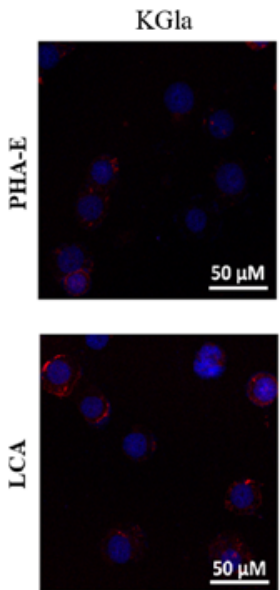

KGla (HS27A)
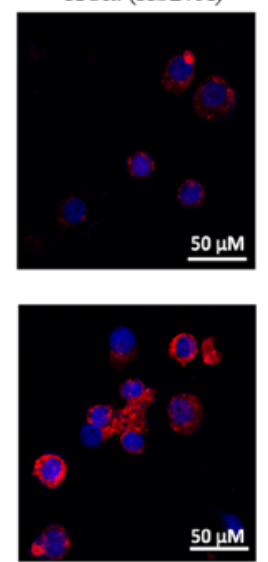

Figure 5. Comparative glycogene expression and differential glycopatterns of KG1a cells vs. co-cultured KG1a cells. (A) Gene expression of FUT8, MGAT2, MGAT3, MGAT4B and MGAT5 was analyzed by real-time PCR in triplicate experiments. Relative expression was analyzed by the $2^{-\triangle \Delta C q}$ method and presented as $\log _{2}$ relative expression for KG1a vs. co-cultured KG1a cells, with $\log _{2}(3 / 2)$ and $\log _{2}(2 / 3)$ as threshold values. Values above $\log _{2}(3 / 2)$ and below $\log _{2}(2 / 3)$ indicate significant upregulation and downregulation, respectively. (B-D) Differential glycopatterns in KG1a and co-cultured KG1a. The four indicated lectins, conjugated with Cy3, were used. (B) Flow cytometric analysis. (C) Quantification of intensity. (D) Lectin staining was performed. The data shown are representative of three independent experiments. ${ }^{*} \mathrm{P}<0.05,{ }^{* * *} \mathrm{P}<0.001$. Error bars, SEM. KG1a (HS27A), co-cultured cells. 
A

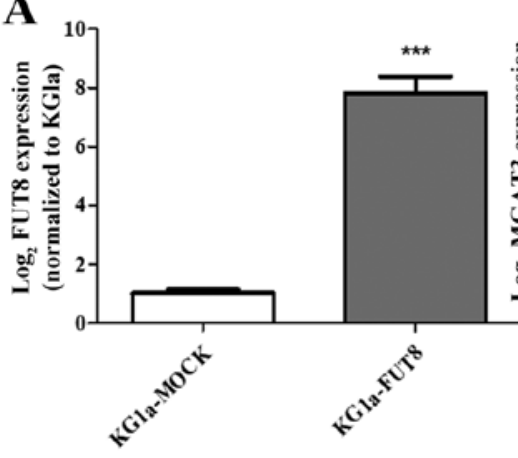

B

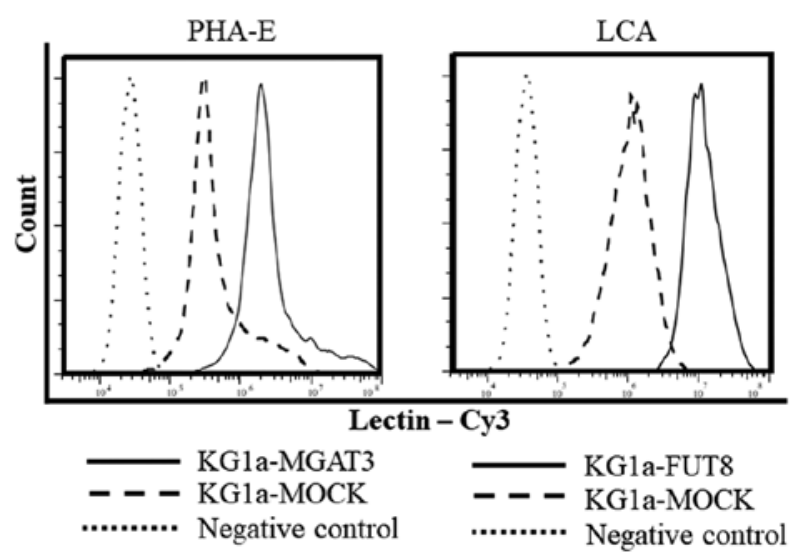

$\mathbf{C}$

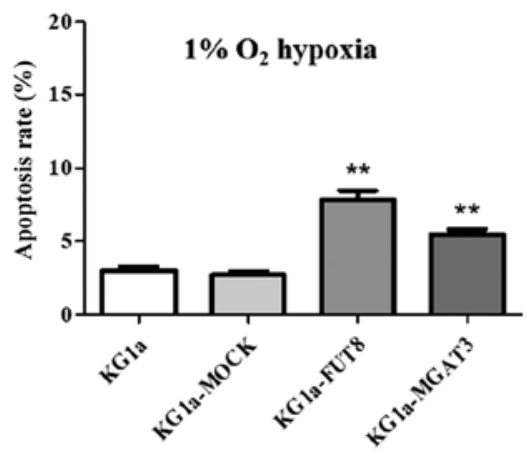

D

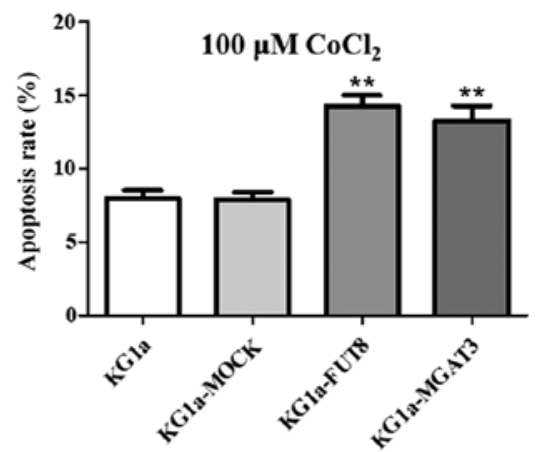

Figure 6. Overexpression of FUT8 and MGAT3 in KGla cells and their apoptosis rate as measured by flow cytometry. (A) Validation of the overexpression of FUT8 and MGAT3 in KG1a cells. (B) Flow cytometric analysis to verify the corresponding bisecting $N$-glycan and core fucosylation in KG1a cells overexpressing MGAT3 and FUT8, respectively. (C and D) Apoptosis rate in $\mathrm{KG}_{1}$ a cells overexpressing $\mathrm{FUT}_{8}$ or $\mathrm{MGAT}_{3}$ under $1 \% \mathrm{O}_{2}$ hypoxia or $100 \mu \mathrm{M} \mathrm{CoCl}_{2}$ by flow cytometry. The data shown are representative of three independent experiments. ${ }^{* *} \mathrm{P}<0.01,{ }^{* * * *} \mathrm{P}<0.001$. Error bars, SEM. KG1a (HS27A), co-cultured cells.

Validation by flow cytometry of $N$-glycan expression differences in co-cultured KGla cells under hypoxic conditions. To verify the function of FUT8 and MGAT3, we overexpressed these two genes in KGla cells (Fig. 6A). Flow cytometric data revealed that the corresponding bisecting $N$-glycan or core fucosylation in KGla cells that overexpressed MGAT3 or FUT8 was enhanced (Fig. 6B). Our data showed an enhanced apoptosis rate in KGla cells that overexpressed MGAT3 or FUT8 under both hypoxic conditions and $\mathrm{CoCl}_{2}$ treatment (Fig. 6C and D). We suggest that modulation of glycogenes may play a role in regulating the apoptosis in KGla cells.

\section{Discussion}

Tumor/stromal cell interactions are frequently part of cell social networks, play essential roles in tumor development and growth, and provide useful therapeutic targets. Our 2010 studies were focused on an in vitro co-culture system involving two stromal cell lines (HS5 and HS27A) derived from a healthy bone marrow donor. Cells from patients with advanced MDS showed increased susceptibility to TNF $\alpha$-induced apoptosis following stromal contact $(8,9)$. Our 2013 in vivo study, based on the in vitro observations, involved injection of HS5 or HS27A cells, together with primary MDS marrow cells, into NOD.Cg-Prkde ${ }^{\text {scid }}$ Il2rg ${ }^{\text {tm1Wjl }}$ (NSG) mice (12). HS27A cells, but not HS5 cells, facilitated the engraftment of clonal MDS cells in vivo. The present study focused on the crosstalk that occurs between hematopoietic and HS27A cells (but not HS5). Among the numerous factors present in the tumor microenvironment, hypoxia is a common feature of myeloid malignancies and has been shown to affect such HSC behaviors as apoptosis, proliferation, differentiation and resistance to chemotherapy. The role of hypoxia and its downstream signaling in hematopoiesis is controversial, since both promoting and inhibitory effects have been reported (39). Velasco-Hernandez et al found that deletion of HIF-1 $\alpha$ in mouse hematopoietic cells promoted acute myeloid leukemia progression (40). Other studies indicated that hypoxia promoted leukemogenic niche metabolism and cytokine secretion $(41,42)$. In the present study, we compared the apoptosis rates of hematopoietic KGla cells alone or co-cultured with HS27A cells under hypoxic conditions. Our data clearly indicated that elevated caspase 8 activities were found in co-cultured KGla cells. However, cleaved caspase-9 had no significant changes in co-cultured KG1a cells, even though the total amount of caspase-9 was increased, indicating that the co-culture may trigger the expression of total caspase- 9 which possibly make the co-cultured KG1a cells becoming more sensitive to cell death signals (43-45). Increased levels of p53 in the co-cultured cells revealed that the apoptotic pathway was p53-dependent.

Functional proteins in both normal and malignant cells are maintained by post-translational modifications (PTMs), which include phosphorylation, ubiquitination, methylation, $\mathrm{N}$-acetylation and glycosylation (30). Glycosylation, the most 
common PTM, is involved in a wide variety of biological processes. Glycosylation of proteins (giving rise to glycoproteins) in eukaryotic cells is classified on the basis of various linkages of glycans to protein core regions e.g., $N$-linked glycan (GlcNAc linkage to Asn), $O$-linked glycan (O-GalNAc linkage to Ser/Thr), and other $O$-linked glycan varieties (e.g., $O$-linked mannose, $O$-linked GlcNAc) (30). Biological processes mediated by glycans include signal transduction, inflammation, virus/bacteria-host interactions, cell-cell interactions and cancer development and progression. Our MS analyses of $\mathrm{N}$-glycan profiles of co-cultured KGla cells under hypoxia revealed increases in bi- and tri-antennary type, GlcNAc type and fucose type $N$-glycans.

Synthesis of $N$-glycans is catalyzed by various enzymes termed glycosyltransferases. Fucosylation is catalyzed by FUT8, which transfers a fucose (Fuc) moiety from GDP- $\beta$-L-Fuc to the innermost GlcNAc residue of an $N$-glycan, resulting in $\alpha 1,6$-Fuc residue as core Fuc. FUT8 activity is upregulated during epithelial-mesenchymal transition (EMT) through transactivation of $\beta$-catenin/lymphoid enhancer-binding factor-1 (LEF-1) (46). The glycosyltransferase MGAT3 catalyzes transfer of GlcNAc to the core $\beta$-mannose residue of $N$-glycans having $\beta 1,4$-linkage. Addition of bisecting GlcNAc residue to core $\beta$-mannose by MGAT3 inhibits activities of mannosidase II and other GlcNAc transferases (MGAT2, MGAT4). Li et al and $\mathrm{Xu}$ et al reported reduced levels of MGAT3 and its product (bisecting $N$-glycans) in a TGF- $\beta 1$-induced EMT model $(16,47)$. Takahashi et al observed that MGAT3 overexpression reduced the amount of poly-N-acetyllactosamine on $N$-glycans of EGFR, indicating that specific terminal structures of $\mathrm{N}$-glycans regulated EGFR endocytosis through interaction with carbohydrate recognition molecules (48). MGAT3 exerts a stabilizing effect on E-cadherin at the cell membrane by inducing a delay in the turnover rate of the protein, thereby contributing to stable and functional adhesion, and blocking clathrin-dependent E-cadherin endocytosis (49). Remodeling of glycans by MGAT3 contributes to the retention of E-cadherin in membrane by mesalamine (5-ASA) (50). In contrast, MGAT5 promotes destabilization of E-cadherin, leading to its mislocalization, formation of unstable junctions and impairment of cell-cell adhesion (49). Inhibition of MGAT5 activity reduced liver fibrosis and suppressed TGF- $\beta 1$-induced EMT in hepatocytes, as evidenced by reversal of EMT markers. Our model (co-cultured KG1a under hypoxia) showed increased expression of FUT8, MGAT3 and MGAT5, but no notable change of MGAT2 or MGAT4B. Increased binding affinity of lectins PHA-E and WGA in our model revealed elevated levels of bisecting-GlcNAc and (GlcNAc) $)_{n}$ structures, in striking contrast to $N$-glycan structures in co-cultured KG1a under normoxia (data not shown). We then overexpressed FUT8 and MGAT3 genes in KG1a cells to ascertain whether modified glycogenes could impact the cell apoptosis of KGla cells under hypoxia. Our data suggested that modified glycogenes of both FUT8 and MGAT3 could enhance cell apoptosis in KG1a cells. Our further studies will focus on the functional role of FUT8 and MGAT3 in primary cells, as well as the microenvironmental niche.

In conclusion, the present study provided the first demonstration of hypoxia-induced apoptosis in KGla hematopoietic cells following contact with HS27A stromal cells, and $\mathrm{N}$-glycan profiles of KGla cells before and after stromal contact under hypoxia. Our findings are useful references for future studies based on co-culture models, and may help elucidate the roles of hematopoietic and stromal cells in bone-related diseases. Our ongoing studies will focus on the functions of $N$-glycans in co-culture systems and the clinical relevance of these $N$-glycans.

\section{Acknowledgements}

The authors are grateful to Dr S. Anderson for the English language editing of the manuscript.

\section{Funding}

The present study was supported in part by grants from the National Natural Science Foundation of China (nos. 81470294, 31400691 and 81770123), the Natural Science Foundation of Jiangsu Province (BK20140169), the Fundamental Research Funds for the Central Universities (JUSRP51619B) and the 13115 Key Projects of Scientific and Technical Innovation of Shaan'xi Province (2010ZDKG-53).

\section{Availability of data and materials}

The datasets used during the present study are available from the corresponding author upon reasonable request.

\section{Authors' contributions}

XL and YW designed the study and wrote the manuscript. XP, SZ, XL, ZT and JG performed the experiments. FG critiqued the manuscript and was also involved in the conception and design of the study. All authors have read and approved the final manuscript. All authors agreed to be accountable for all aspects of the work in ensuring that questions related to the accuracy or integrity of any part of the work are appropriately investigated and resolved.

\section{Ethics approval and consent to participate}

Not applicable.

\section{Patient consent for publication}

Not applicable.

\section{Competing interests}

The authors declare that they have no competing interests.

\section{References}

1. Trentin JJ: Determination of bone marrow stem cell differentiation by stromal hemopoietic inductive microenvironments (HIM). Am J Pathol 65: 621-628, 1971.

2. Bennett $\mathrm{M}$ and Kumar V: 89Sr-induced bone marrow aplasia: Effects on seed (stem cells) and soil (inductive microenvironment). Lab Invest 49: 235-236, 1983

3. Morrison SJ and Scadden DT: The bone marrow niche for haematopoietic stem cells. Nature 505: 327-334, 2014.

4. Simmons PJ and Torok-Storb B: Identification of stromal cel precursors in human bone marrow by a novel monoclonal antibody, STRO-1. Blood 78: 55-62, 1991. 
5. Hanahan D and Weinberg RA: Hallmarks of cancer: The next generation. Cell 144: 646-674, 2011.

6. Manabe A, Coustan-Smith E, Behm FG, Raimondi SC and Campana D: Bone marrow-derived stromal cells prevent apoptotic cell death in B-lineage acute lymphoblastic leukemia. Blood 79: 2370-2377, 1992.

7. Ayala F, Dewar R, Kieran M and Kalluri R: Contribution of bone microenvironment to leukemogenesis and leukemia progression. Leukemia 23: 2233-2241, 2009.

8. Marcondes AM, Li X, Gooley TA, Milless B and Deeg HJ: Identification of DJ-1/PARK-7 as a determinant of stroma-dependent and TNF-alpha-induced apoptosis in MDS using mass spectrometry and phosphopeptide analysis. Blood 115: 1993-2002, 2010.

9. Li X, Marcondes AM, Gooley TA and Deeg HJ: The helix-loop-helix transcription factor TWIST is dysregulated in myelodysplastic syndromes. Blood 116: 2304-2314, 2010.

10. Marcondes AM, Li X, Tabellini L, Bartenstein M, Kabacka J, Sale GE, Hansen JA, Dinarello CA and Deeg HJ: Inhibition of IL-32 activation by $\alpha-1$ antitrypsin suppresses alloreactivity and increases survival in an allogeneic murine marrow transplantation model. Blood 118: 5031-5039, 2011.

11. Li X, Xu F, Chang C, Byon J, Papayannopoulou T, Deeg HJ and Marcondes AM: Transcriptional regulation of miR-10a/b by TWIST-1 in myelodysplastic syndromes. Haematologica 98 : 414-419, 2013

12. Li X, Marcondes AM, Ragoczy T, Telling A and Deeg HJ: Effect of intravenous coadministration of human stroma cell lines on engraftment of long-term repopulating clonal myelodysplastic syndrome cells in immunodeficient mice. Blood Cancer J 3: e113, 2013.

13. Li X and Deeg HJ: Murine xenogeneic models of myelodysplastic syndrome: An essential role for stroma cells. Exp Hematol 42: 4-10, 2014

14. Kerbauy DM, Lesnikov V, Torok-Storb B, Bryant E and Deeg HJ: Engraftment of distinct clonal MDS-derived hematopoietic precursors in NOD/SCID-beta2-microglobulin-deficient mice after intramedullary transplantation of hematopoietic and stromal cells. Blood 104: 2202-2203, 2004.

15. Li M, Song L and Qin X: Glycan changes: Cancer metastasis and anti-cancer vaccines. J Biosci 35: 665-673, 2010.

16. Li X, Wang X, Tan Z, Chen S and Guan F: Role of glycans in cancer cells undergoing epithelial-mesenchymal transition. Front Oncol 6: 33, 2016.

17. Dube DH and Bertozzi CR: Glycans in cancer and inflammationpotential for therapeutics and diagnostics. Nat Rev Drug Discov 4: 477-488, 2005

18. Groux-Degroote S, Guérardel Y and Delannoy P: Gangliosides: Structures, biosynthesis, analysis, and roles in cancer. Chembiochem 18: 1146-1154, 2017.

19. Timoshenko AV, Lanteigne J and Kozak K: Extracellular stress stimuli alter galectin expression profiles and adhesion characteristics of HL-60 cells. Mol Cell Biochem 413: 137-143, 2016.

20. Le Marer N: GALECTIN-3 expression in differentiating human myeloid cells. Cell Biol Int 24: 245-251, 2000.

21. Wan SY, Zhang TF and Ding Y: Galectin-3 enhances proliferation and angiogenesis of endothelial cells differentiated from bone marrow mesenchymal stem cells. Transplant Proc 43 3933-3938, 2011.

22. Mohyeldin A, Garzón-Muvdi T and Quiñones-Hinojosa A: Oxygen in stem cell biology: A critical component of the stem cell niche. Cell Stem Cell 7: 150-161, 2010.

23. Wenger RH: Cellular adaptation to hypoxia: O2-sensing protein hydroxylases, hypoxia-inducible transcription factors, and O2-regulated gene expression. FASEB J 16: 1151-1162, 2002.

24. Semenza GL: Intratumoral hypoxia, radiation resistance, and HIF-1. Cancer Cell 5: 405-406, 2004.

25. Semenza GL: Targeting HIF-1 for cancer therapy. Nat Rev Cancer 3: 721-732, 2003.

26. Danet GH, Pan Y, Luongo JL, Bonnet DA and Simon MC Expansion of human SCID-repopulating cells under hypoxic conditions. J Clin Invest 112: 126-135, 2003.

27. Li X, Wan T, Zhang S, Li D and Han X: Quantitative proteomic analysis and comparison of two bone marrow stromal cell lines using the SILAC method. Exp Hematol 44: 1059-1071, 2016.

28. Li X, Li D, Pang X, Yang G, Deeg HJ and Guan F: Quantitative analysis of glycans, related genes, and proteins in two human bone marrow stromal cell lines using an integrated strategy. Exp Hematol 43: 760-769.e7, 2015.

29. Livak KJ and Schmittgen TD: Analysis of relative gene expression data using real-time quantitative PCR and the $2^{-\Delta \Delta C_{\mathrm{T}}}$ method. Methods 25: 402-408, 2001.
30. Tan Z,Lu W, Li X, Yang G, Guo J, Yu H, Li Z and Guan F: Altered $\mathrm{N}$-Glycan expression profile in epithelial-to-mesenchymal transition of NMuMG cells revealed by an integrated strategy using mass spectrometry and glycogene and lectin microarray analysis. J Proteome Res 13: 2783-2795, 2014

31. Guan F, Tan Z, Li X, Pang X, Zhu Y, Li D and Yang G: A lectin-based isolation/enrichment strategy for improved coverage of N-glycan analysis. Carbohydr Res 416: 7-13, 2015.

32. Ying SX, Seal S, Abbassi N, Hockenbery DM, Kiem HP, Li X, Pagel JM, Gopal AK and Deeg HJ: Differential effects of bexarotene on intrinsic and extrinsic pathways in TRAIL-induced apoptosis in two myeloid leukemia cell lines. Leuk Lymphoma 48: 1003-1014, 2007

33. de Thonel A, Bettaïeb A, Jean C, Laurent G and Quillet-Mary A: Role of protein kinase $\mathrm{C}$ zeta isoform in Fas resistance of immature myeloid KG1a leukemic cells. Blood 98: 3770-3777, 2001.

34. Lopez-Sánchez LM, Jimenez C, Valverde A, Hernandez V, Peñarando J, Martinez A, Lopez-Pedrera C, Muñoz-Castañeda JR De la Haba-Rodríguez JR, Aranda E and Rodriguez-Ariza A: $\mathrm{CoCl}_{2}$, a mimic of hypoxia, induces formation of polyploid giant cells with stem characteristics in colon cancer. PLoS One 9: e99143, 2014.

35. Guan F, Schaffer L, Handa K and Hakomori SI: Functional role of gangliotetraosylceramide in epithelial-to-mesenchymal transition process induced by hypoxia and by TGF-\{beta\}. FASEB J 24: 4889-4903, 2010.

36. Ohtsuka T, Ryu H, Minamishima YA, Macip S, Sagara J, Nakayama KI, Aaronson SA and Lee SW: ASC is a Bax adaptor and regulates the p53-Bax mitochondrial apoptosis pathway. Nat Cell Biol 6: 121-128, 2004.

37. Marchenko ND and Moll UM: Mitochondrial death functions of p53. Mol Cell Oncol 1: e955995, 2014.

38. Etxebarria J and Reichardt NC: Methods for the absolute quantification of N-glycan biomarkers. Biochim Biophys Acta 1860: $1676-1687,2016$.

39. Korn $\mathrm{C}$ and Méndez-Ferrer S: Myeloid malignancies and the microenvironment. Blood 129: 811-822, 2017.

40. Velasco-Hernandez T, Hyrenius-Wittsten A, Rehn M, Bryder D and Cammenga J: HIF- $1 \alpha$ can act as a tumor suppressor gene in murine acute myeloid leukemia. Blood 124: 3597-3607, 2014.

41. Drolle H, Wagner M, Vasold J, Kütt A, Deniffel C, Sotlar K, Sironi S, Herold T, Rieger C and Fiegl M: Hypoxia regulates proliferation of acute myeloid leukemia and sensitivity against chemotherapy. Leuk Res 39: 779-785, 2015.

42. Kuschel A, Simon P and Tug S: Functional regulation of HIF-1a under normoxia-is there more than post-translational regulation? J Cell Physiol 227: 514-524, 2012.

43. Liedtke C Gröger N, Manns MP and Trautwein C: Interferon-alpha enhances TRAIL-mediated apoptosis by up-regulating caspase- 8 transcription in human hepatoma cells. J Hepatol 44: 342-349, 2006.

44. Ruiz-Ruiz C, Ruiz de Almodóvar C, Rodríguez A, Ortiz-Ferrón G, Redondo JM and López-Rivas A: The up-regulation of human caspase- 8 by interferon-gamma in breast tumor cells requires the induction and action of the transcription factor interferon regulatory factor-1. J Biol Chem 279: 19712-19720, 2004.

45. Gomyo Y, Sasaki J, Branch C, Roth JA and Mukhopadhyay T: 5-aza-2'-deoxycytidine upregulates caspase-9 expression cooperating with p53-induced apoptosis in human lung cancer cells. Oncogene 23: 6779-6787, 2004.

46. Chen CY, Jan YH, Juan YH, Yang CJ, Huang MS, Yu CJ, Yang PC, Hsiao M, Hsu TL and Wong CH: Fucosyltransferase 8 as a functional regulator of nonsmall cell lung cancer. Proc Natl Acad Sci USA 110: 630-635, 2013.

47. Xu Q, Isaji T, Lu Y, Gu W, Kondo M, Fukuda T, Du Y and Gu J: Roles of $\mathrm{N}$-acetylglucosaminyltransferase III in epithelial-to-mesenchymal transition induced by transforming growth factor $\beta 1$ (TGF- $\beta 1$ ) in epithelial cell lines. J Biol Chem 287: 16563-16574, 2012.

48. Takahashi M, Kizuka Y, Ohtsubo K, Gu J and Taniguchi N: Disease-associated glycans on cell surface proteins. Mol Aspects Med 51: 56-70, 2016 .

49. Pinho SS, Figueiredo J, Cabral J, Carvalho S, Dourado J, Magalhães A, Gärtner F, Mendonfa AM, Isaji T, Gu J, et al: E-cadherin and adherens-junctions stability in gastric carcinoma: Functional implications of glycosyltransferases involving N-glycan branching biosynthesis, $\mathrm{N}$-acetylglucosaminyltransferases III and V. Biochim Biophys Acta 1830: 2690-2700, 2013.

50. Khare V, Lang M, Dammann K, Campregher C, Lyakhovich A and Gasche C: Modulation of N-glycosylation by mesalamine facilitates membranous E-cadherin expression in colon epithelial cells. Biochem Pharmacol 87: 312-320, 2014 